Ann. Génét. Sél. anim., I972, 4 (3), 4II-42I.

\title{
ÉQUILIBRES STABLES \\ OU AVEC OSCILLATIONS PÉRIODIQUES \\ DES FRÉQUENCES ALLÉLIQUES EN SÉLECTION PAR TEST DE DESCENDANCE : EXEMPLES NUMÉRIQUES
}

\author{
B. POUJARDIEU et P. MÉRAT \\ Laboratoire de Méthodologie génétique, \\ Centre de Recherches de Toulouse, I. N. R. A., \\ B. P. 12, 31 - Castanet-Tolosan \\ Laboratoire de Génétique factorielle, \\ Centre national de Recherches zootechniques, I. N. R. A., \\ 78 - Jouy-en-Josas
}

\section{RÉSUMÉ}

Sur une série d'exemples numériques, nous avons suivi l'évolution, au cours de ${ }_{5}$ générations de sélection pour un caractère quantitatif, de la fréquence d'un gène $A$ à un locus correspondant à deux allèles présentant une surdominance pour ce caractère, avec une variation " résiduelle " distribuée normalement. La sélection est supposée être exercée dans un seul sexe par test de descendance avec un nombre relativement grand de représentants de l'autre sexe, les générations étant séparées dans le temps et les reproducteurs appariés au hasard.

Il y a toujours maintien des deux allèles existant au départ, et, dans la majorité des cas, on tend vers une fréquence unique stable pour A. On vérifie d'autre part, dans certaines situations, la possibilité d'oscillations permanentes de cette fréquence. Ces oscillations sont d'autant plus sensibles que l'intensité de sélection est plus grande, que les familles sont plus nombreuses et que la supériorité du génotype hétérozygote est plus marquée. Cependant, les fluctuations de la moyenne générale de la population qui en résultent sont souvent d'une importance limitée.

\section{INTRODUCTION}

Dans un article antérieur, l'un de nous (MÉRAT, I970) avait examiné l'effet, pour un caractère quantitatif, d'une sélection par familles (test de descendance, test sur frères et sœurs ou sélection récurrente) sur l'évolution de la fréquence d'un 
gène présentant des différences moyennes entre génotypes pour ce caractère. Le cas d'un locus à deux allèles avec "surdominance " (supériorité de l'hétérozygote) avait été analysé, en comparant notamment le rôle de l'intensité de sélection suivant l'importance des différences entre génotypes au locus considéré.

La possibilité d'oscillations, momentanées ou "entretenues ", des fréquences alléliques lors d'un choix par test de descendance avait été signalée. Toutefois, si cette possibilité est démontrable par un raisonnement théorique, ce n'est que par tâtonnements que 1'on peut déterminer, dans des cas concrets, les conditions de réalisation de ce phénomène et les valeurs exactes entre lesquelles oscillera la fréquence d'un gène. L'objet du présent travail est d'apporter ces précisions en analysant des résultats numériques calculés à l'aide d'un ordinateur.

\section{I. - POSITION DU PROBLİ⿴囗M}

Reprenant les hypothèses du premier article déjà cité, nous supposerons que, relativement à un caractère quantitatif $\mathrm{X}$, une sélection est opérée sur des mâles d'après la moyenne de leur descendance, d'effectif constant, obtenue pour chacun avec un nombre égal et relativement grand de femelles, aucune sélection n'étant faite dans le sexe femelle.

Nous nous limiterons au cas d'une population nombreuse (modèle déterministe) d'un organisme diploïde à reproduction sexuée, avec générations séparées et appariement au hasard des reproducteurs des deux sexes. Nous considérons un locus à deux allèles A et $a$ avec "surdominance " et supposons qu'en dehors de l'effet de ce locus les distributions résiduelles pour le caractère en question sont normales et de même variance constante (ce qui n'est qu'approximatif après une ou plusieurs générations de sélection).

Le choix des reproducteurs revient à un tri par troncature dans la distribution produit des moyennes de descendances relatives à chaque génotype de parent mâle.

Nous désignerons par $d$ et $d^{\prime}$ (exprimés en écarts-réduits des distributions " résiduelles " des individus) respectivement l'excès de la valeur moyenne des individus AA et des A $a$ sur celle des $a a\left(d^{\prime}>d\right)$, par $\mathrm{N}$ l'effectif, supposé égal, des descendances de chaque père.

Nous avions indiqué (MÉRAT, I970) l'expression des espérances mathématiques des valeurs moyennes et des variances de la descendance en testage, respectivement, des reproducteurs AA, Aa et $a a$. Cette expression, reproduite plus loin (tab1. I) montre que le changement de fréquence de $\mathrm{A}$ en une génération modifie non seulement les proportions respectives des distributions, mais aussi leurs moyennes et leurs variances, au moins pour un gène " majeur ".

\section{II. - MÉTHODE DE CALCUI,} devons

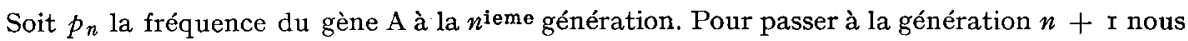
Io Choisir par progeny-test les géniteurs mâles, c'est-à-dire calculer, pour chaque distribution 
par génotype de père, la position du point de troncature et la proportion d'individus retenue.

$2^{\circ}$ Calculer la fréquence de $\mathrm{A}$ à la génération $n+\mathbf{I}$ en fonction des proportions de pères retenus par génotype.

\section{I. - Choix des géniteurs mâles}

Sera retenu comme géniteur mâle tout père dont la moyenne des descendants sera supérieure à une valeur $y$ telle que la fréquence des descendances de moyenne supérieure ou égale à $y$ soit égale à une constante $v$, proportion de géniteurs mâles à conserver. (tabl. I).

La population des descendants utiles pour la sélection est définie par le tableau ci-dessous

\section{TABLEAU I}

Paramètres caractérisant la population des descendants après une génération de sélection (Locus avec surdominance; sélection par test de descendance dans un sexe)

\begin{tabular}{|c|c|c|c|}
\hline $\begin{array}{l}\text { Génotype } \\
\text { du père }\end{array}$ & Fréquence & $\begin{array}{c}\text { Moyenne } \\
\text { des descendants }\end{array}$ & Variance des descendants \\
\hline $\begin{array}{l}\mathrm{AA} \\
\mathrm{A} a \\
a a\end{array}$ & $\begin{array}{l}p_{n}^{2} \\
2 p_{n}\left(1-p_{n}\right) \\
\left(1-p_{n}\right)^{2}\end{array}$ & $\begin{array}{l}m_{1}=p_{n}\left(d-d^{\prime}\right)+d^{\prime} \\
m_{2}=\left(p d+d^{\prime}\right) / 2 \\
m_{3}=p d^{\prime}\end{array}$ & $\begin{array}{l}\sigma_{1}^{2}=\left[p_{n}\left(1-p_{n}\right)\left(d-d^{\prime}\right)^{2}+1\right] / \mathrm{N} \\
\sigma_{2}^{2}=\left[p_{n}\left(1-p_{n}\right) d^{2} / 2+\left(d^{\prime}-d p\right)^{2} / 4+1\right] / \mathrm{N} \\
\sigma_{3}^{2}=\left[p_{n}\left(1-p_{n}\right) d^{\prime 2}+1\right] / \mathrm{N}\end{array}$ \\
\hline Total & 1 & $p\left[2 d^{\prime}-p\left(2 d^{\prime}-d\right)\right]$ & \\
\hline
\end{tabular}

Par application du théorème central limite aux moyennes de chaque descendance de femelles de génotype donné, accouplées à chaque père, nous pouvons assimiler les distributions. des moyennes des descendances, par type génétique de père, à des distributions normales $\mathrm{N}\left(m_{i}, \sigma_{i}\right)$

Le problème consiste alors à calculer $y$ tel que

$$
p_{n}^{2} \int_{y}^{+\infty} f_{1}(t) \mathrm{d} t+2 p_{n}\left(\mathrm{I}-p_{n}\right) \int_{y}^{+\infty} f_{2}(t) \mathrm{d} t+\left(\mathrm{I}-p_{n}\right)^{2} \int_{y}^{+\infty} f_{3}(t) \mathrm{d} t=v
$$

avec $f_{i}(t)=\frac{\mathbf{I}}{\sqrt{2 \pi} \sigma_{i}} \mathrm{e}^{-\frac{\left(t-m_{i}\right)^{2}}{2 \sigma_{i}^{2}}}$, distribution normale résiduelle.

En posant $u=\frac{t-m_{i}}{\sigma_{i}}$ nous obtenons $g(u) \mathrm{d} u=\frac{\mathbf{I}}{\sqrt{2 \pi}} \mathrm{e}^{-\frac{u^{2}}{2}} \mathrm{~d} u$. Le calcul revient alors à rechercher les valeurs $u_{1}, u_{2}, u_{3},\left(u_{i}=\frac{y-m_{i}}{\sigma_{i}}\right)$ telles que :

$$
p_{n}^{2} s_{1}+2 p_{n}\left(\mathbf{I}-p_{n}\right) s_{2}+\left(\mathrm{I}-p_{n}\right)^{2} s_{3}=v
$$

$\operatorname{avec} s_{i}=\int_{u i}^{+\infty} g(u) \mathrm{d} u$.

A cet effet, nous avons fait l'approximation de l'intégrale d'une loi normale centrée réduite, par l'algorithme suivant (HAstings, r955) :

et

$$
\int_{u i}^{+\infty} g(u) \mathrm{d} u=\frac{\mathrm{I}}{2}-\int_{0}^{u i} g(u) \mathrm{d} u
$$

$$
\left.\left.\varphi(u i)=\int_{0}^{u_{1}} g(u) \mathrm{d} u=\frac{\mathrm{I}}{2}\left[\mathrm{I}-\frac{\mathrm{I}}{\left[\sum_{j=0}^{5} a_{j}\left(\frac{u_{i}}{\sqrt{2}}\right)^{\mathrm{j}}\right.}\right]\right]^{8}\right] \text { pour } u_{1} \geqslant 0
$$




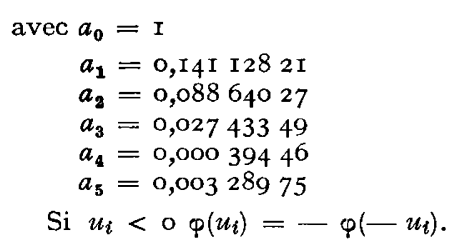

Pour calculer les valeurs de $s_{1}, s_{2}$ et $s_{3}$, nous avons procédé par approximations successives de $y$ à partir d'une valeur $y=m_{2}$ jusqu'à ce que la différence entre la valeur calculée $\widehat{v}$ de la fréquence des familles globalement gardées et la valeur imposée $v$ soit inférieure ou égale en valeur absolue à $5 \cdot 10^{-5}$.

Le pas de variation initial de $y$ est de $0 . \mathbf{I} / \sigma_{2}$; il est réduit de moitié chaque fois que nous obtenons deux valeurs consécutives de signe contraire de la différence $\left(v-\tau^{\prime}\right)$. Dans le cas où à la fin de 20 ooo itérations $|v-\widehat{v}|>5 \cdot 10^{-5}$ nous avons laissé le problème sans solution.

L'erreur maximum faite sur l'intégrale est de $\mathbf{1 O}^{-5}$. La fonction erreur est périodique et son amplitude diminue lorsque $u_{i}$ augmente.

\section{2. - Calcul de $p_{n+1}$}

Puisque la population de femelles n'est pas sélectionnée $p_{n+1}=\frac{\mathrm{I}}{2}\left(p_{n}^{\prime}+p_{n}\right)$, $p_{n}^{\prime}$ étant la fréquence de $\mathrm{A}$ parmi les mâles retenus, d'où

$$
\begin{aligned}
& p_{n+1}=\frac{\mathrm{I}}{2}\left[\frac{p_{n}^{2} s_{1}+p_{n}\left(\mathrm{I}-p_{n}\right) s_{2}}{p_{n}^{2} s_{1}+2 p_{n}\left(\mathrm{I}-p_{n}\right) s_{2}+\left(\mathrm{I}-p_{n}\right)^{2} s_{3}}+p_{n}\right] \\
& p_{n+1}=\frac{\mathrm{I}}{2} p_{n} \frac{p_{n}\left(\mathrm{I}+p_{n}\right) s_{1}+\left(\mathrm{I}+2 p_{n}\right)\left(\mathrm{I}-p_{n}\right) s_{2}+\left(\mathrm{I}-p_{n}\right)^{2} s_{3}}{p_{n}^{2} s_{1}+2 p n\left(\mathrm{I}-p_{n}\right)} \frac{\varsigma_{2}+\left(\mathrm{I}-p_{n}\right)^{2} s_{3}}{}
\end{aligned}
$$

quantité calculée sous la forme :

$$
p_{n+1}=p_{n}\left\{p_{n}\left[p_{n}\left(s_{1}-2 s_{2}+s_{3}\right)+s_{1}+s_{2}-2 s_{3}\right]+s_{3}+s_{3}\right\} / 2 v
$$

\section{3. - Définition du programme et utilisation des résultats}

Pour calculer les paramètres de chaque génération, nous avons écrit en ForTRAN IV sur IBM 360-50 un programme.

Une situation de calcul est définie par 5 paramètres :

r. La fréquence originelle de $\mathrm{A}$, prise entre o, I et 0,9 si $d \neq 0$ entre o, I et o,4 si $d=0$

puisque, pour cette dernière valeur de $d$, le graphe de la fonction $p_{n_{+1}}=\psi\left(p_{n}\right)$ est symétrique par rapport à $\mathrm{I} / 2$.

2. Une combinaison de $d$ et $d^{\prime}$; les valeurs suivantes ont été choisies :

$$
\begin{aligned}
& d=0 ; 0,5 ; \mathrm{I} \\
& d^{\prime}=0,5 ; \mathrm{I} ; \mathrm{I}, 5
\end{aligned}
$$

3. Une taille de famille de père $(25,50$ ou roo descendants).

4. La proportion d'individus retenus : 5 p. I00; Io p. I00 ; 20 p. I00 ; 50 p. Iоo.

Les calculs ont été effectués sur calculateur IBM 360-50. Pour chaque ensemble de valeurs données aux paramètres précédents, ils ont été poursuivis pendant i 5 générations successives.

Les résultats obtenus comprennent:

- Par génération :

Io La fréquence $p_{n}$ de A ainsi que la fréquence de A parmi les géniteurs mâles.

$2^{\circ}$ La moyenne générale de la population. 
$3^{\circ}$ Par type génétique de père ( $\left.\mathrm{AA}, \mathrm{A} a, a a\right)$ :

- moyenne et variance des familles de même père

- valeurs de $u_{i}$

- valeurs de $s_{i}$

$=$ la différentielle de sélection $\frac{\mathrm{e}^{-\frac{u_{t}^{2}}{2}}}{s_{i}}$

- Sur l'ensemble des 15 générations un graphique récapitulant l'évolution de $p_{n}$.

\section{III. — RÉSULTATS}

Il est bien entendu impossible de présenter le détail de tous les résultats. Nous nous contenterons d'en indiquer les caractéristiques générales les plus typiques.

$$
\text { I. - Équilibres }
$$

Partout, il y a maintien des deux allèles existant au départ, et 1'on tend, soit vers une fréquence stable pour $A$, soit vers l'oscillation de cette fréquence entre deux valeurs, d'une génération à la suivante. Le tableau 2 indique le résultat obtenu dans chaque situation.

Chaque fois qu'il y a tendance vers une fréquence stable, cette fréquence est, comme prévu, égale à $\frac{d^{\prime}}{2 d^{\prime}-d}$ pour $v=0,50$. Elle en est légèrement distincte dans les autres cas, et ce d'autant plus que la taille des familles est plus petite, ceci résultant de la valeur un peu inégale des variances $\sigma_{2}^{1}, \sigma_{2}^{2}$ et $\sigma_{3}^{2}$.

Lorsqu'il y a oscillation de $p$ entre deux valeurs sur deux générations successives, la moyenne de ces deux valeurs est proche également, de $\frac{d^{\prime}}{2 d^{\prime}-d}$.

\section{2. - Oscillations}

Dans une certaine proportion des cas étudiés ici apparaissent, non pas des variations à sens unique de la fréquence $p$, mais des oscillations, soit temporaires, limitées en général aux 3 à 4 premières générations, soit à long terme, ces dernières étant " amorties " (amplitude diminuant graduellement) ou " entretenues " (amplitude ne décroissant pas).

Le tableau 2 donne déjà une indication partielle dans ce sens, relative à l'état obtenu au bout de $\mathrm{I} 5$ générations.

Le tableau suivant (tabl. 3) résume le sens des phénomènes observés, en désignant respectivement par $\mathrm{T}, \mathrm{A}$ et $\mathrm{E}$ les oscillations temporaires, amorties et entretenues (ou à la limite amorties très lentement), avec dans ce dernier cas l'indication des deux fréquences limites entre lesquelles varie $p$.

Dans l'ensemble, ces oscillations sont d'autant plus sensibles que le pourcentage de reproducteurs gardés est plus faible (intensité de sélection plus grande), que les familles sont plus nombreuses, enfin que la supériorité du génotype hétérozygote est plus grande, la différence entre homozygotes étant nulle ou modérée ( $d^{\prime}$ grand en valeur absolue et par rapport à $d$ ). 
B. POUJARDIEU, P. MÉRAT

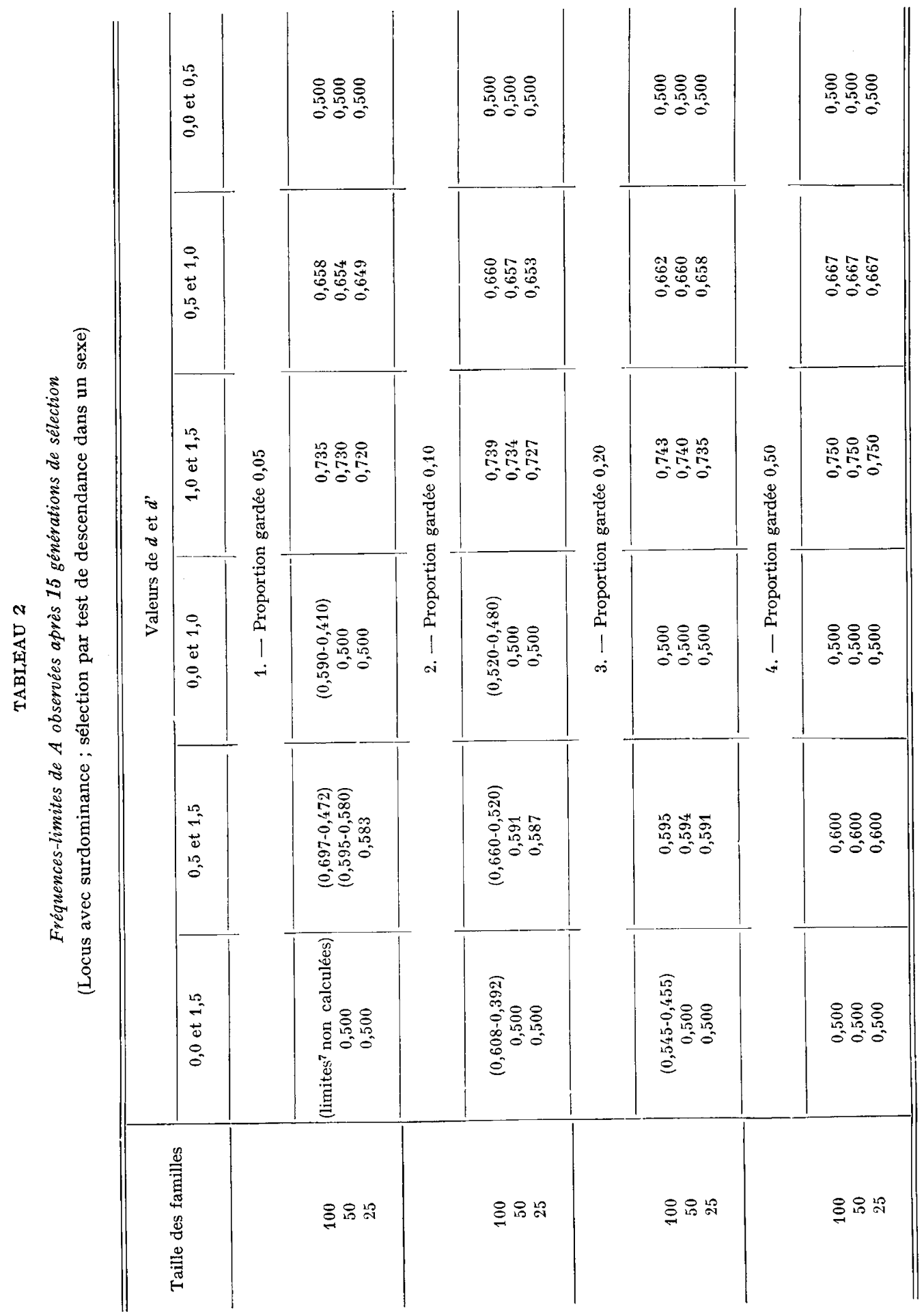


OSCILLATIONS DES FRÉQUENCES ALLÉLIQUES

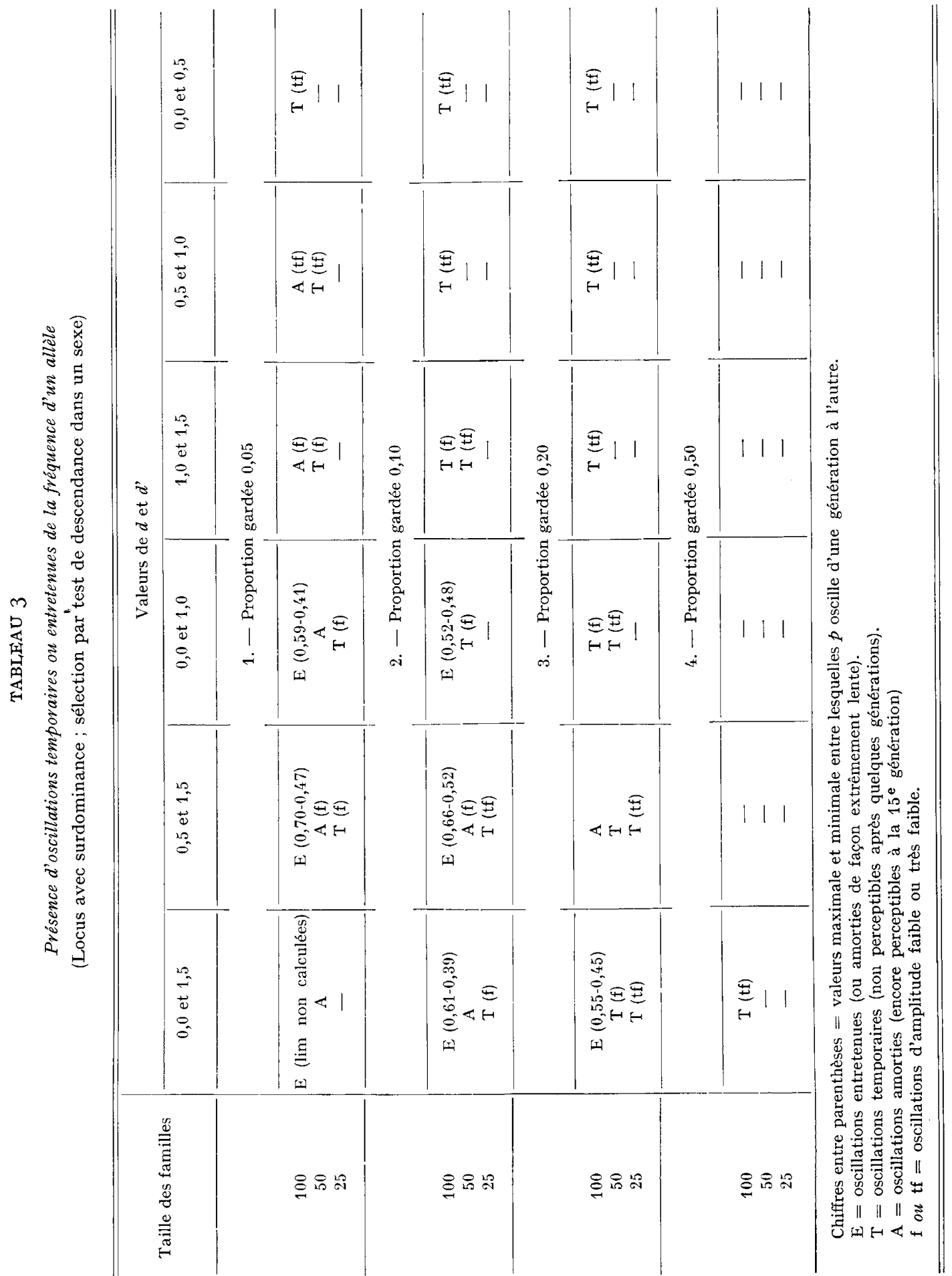

Annales de Génétique animale. - I972. 
Les oscillations que nous appelons " entretenues " ont une amplitude relativement importante dans quelques cas, comme l'indiquent les valeurs minimale et maximale de $p$. La partie I du tableau 4 montre, dans un cas de ce type, l'évolution de $p$ au cours des I5 générations étudiées.

Les oscillations "temporaires " n'ont une amplitude appréciable, presque toujours, que dans les toutes premières générations. Par la suite, ou bien leur amplitude décroît régulièrement, ou bien elles disparaissent entièrement. Les parties 2 et 3 du tableau 4 en représentent des exemples.

Parfois, enfin, on observe un phénomène d'apparence inverse de celui des oscillations " amorties ": il s'agit en fait d'oscillations " entretenues " qui ne s'établissent qu'après plusieurs générations. La partie 4 du tableau 4 en est une illustration.

TABI,EAU 4

Exemples d'évolution de p au cours de 15 générations de sélection

\begin{tabular}{|c|c|c|c|c|}
\hline \multirow[b]{2}{*}{ Génération } & \multicolumn{4}{|c|}{ Valeurs des paramètres et type d'évolution } \\
\hline & $\begin{array}{l}\text { 1. Oscillations } \\
\text { " entretenues" } \\
\begin{array}{c}(v=0,05 ; \mathrm{N}=100 \\
d=0,5 ; d^{\prime}=1,5 \\
p \text { initial }=0,4)\end{array}\end{array}$ & $\begin{array}{l}\because \text {. Oscillations } \\
\text { " amorties " } \\
\begin{array}{c}(v=0,10 ; \mathrm{N}=50 \\
d=0,0 ; d^{\prime}=1,5 \\
p \text { initial }=0,2)\end{array}\end{array}$ & $\begin{array}{l}\text { 3. Oscillations } \\
\text { "temporaires " } \\
\begin{array}{c}(v=0,05 ; \mathrm{N}=25 \\
d=0,5 ; d^{\prime}=1,5 \\
p \text { initial }=0,2)\end{array}\end{array}$ & $\begin{array}{l}\text { 4. Etablissement } \\
\text { progressif } \\
\text { d'oscillations } \\
\text { entretenues } \\
\begin{array}{c}(v=0,05 ; N=100 \\
d=0,5 ; d^{\prime}=1,5 \\
\text { pinitial }=0,6)\end{array}\end{array}$ \\
\hline 1 & 0,4000 & 0,2000 & 0,2000 & 0,6000 \\
\hline 2 & 0,6877 & 0,4462 & $0,,^{\prime} 9^{\prime} 7$ & 0,5776 \\
\hline 3 & 0,4753 & 0,5423 & 0,6058 & 0,6136 \\
\hline 4 & 0,6961 & 0,4655 & 0,5758 & 0,5555 \\
\hline 5 & 0,4719 & 0,5286 & 0,5852 & $0,6 \pm 46$ \\
\hline 6 & 0,6965 & 0,4760 & $0,582 \longleftarrow$ & 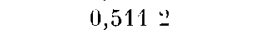 \\
\hline 7 & 0,4718 & 0,5203 & 0,5832 & $0,68+2$ \\
\hline 8 & 0,6965 & 0,4828 & 0,5829 & 0,4770 \\
\hline 9 & 0,4717 & 0,5147 & 0,5830 & 0,6958 \\
\hline 10 & 0,6965 & 0,4874 & 0,5829 & 0,4720 \\
\hline 11 & 0,4717 & 0,5108 & 0,5829 & 0,6965 \\
\hline 12 & 0,6956 & 0,4908 & 0,5829 & $0,471 \&$ \\
\hline 13 & 0,4717 & 0,5079 & 0,5829 & 0,6965 \\
\hline 14 & 0,6965 & 0,4932 & 0,5829 & 0,4717 \\
\hline 15 & 0,4717 & 0,5059 & 0,5829 & 0,6965 \\
\hline
\end{tabular}

\section{IV. - DISCUSSION ET CONCLUSIONS}

\section{I. - Réalisation d'équilibres et leur stabilité}

Les résultats numériques obtenus confirment et précisent ce que le raisonnement théorique faisait prévoir, du point de vue de la réalisation d'équilibres et de leur stabilité. 
En effet, pour qu'il y ait équilibre, il faut et il suffit que $p_{n_{+1}}=p_{n}$ c'est-à-dire que la quantité

$$
\begin{aligned}
2 p_{n}\left[p_{n}^{2} s_{1}+2 p_{n}\left(\mathrm{I}-p_{n}\right) s_{2}+\left(\mathrm{I}-p_{n}^{2}\right)^{2} s_{3}\right] & \\
& -\left[p_{n}^{2}(p+\mathrm{I}) s_{1}+p(\mathrm{I}-p)(2 p+\mathrm{I}) s_{2}+p(\mathrm{I}-p)^{2} s_{3}\right] \equiv 0
\end{aligned}
$$

équation satisfaite si

$$
p(\mathrm{I}-p)\left[p\left(s_{1}-2 s_{2}+s_{3}\right)+s_{2}-s_{3}\right] \equiv 0
$$

Cette identité est vérifiée si $p=$ o ou $p=\mathrm{I}$ (situation sans intérêt) ou si

$$
\left\{\begin{array}{l}
s_{1}-2 s_{2}+s_{3}=0 \\
s_{2}=s_{3}
\end{array}\right\} \rightarrow s_{1}=s_{2}=s_{3}
$$

Pour que $s_{1}=s_{2}=s_{3}$ il faut et il suffit que $u_{1}=u_{2}=u_{3}$ c'est-à-dire

relations vérifiées si

$$
\frac{y-m_{1}}{\sigma_{1}}=\frac{y-m_{2}}{\sigma_{2}}=\frac{y-m_{3}}{\sigma_{3}},
$$

a)

b)

$$
\begin{gathered}
m_{1}=m_{2}=m_{3} \text { et } \sigma_{1}=\sigma_{2}=\sigma_{3} \\
m_{1}=m_{2}=m_{3}=y \\
m_{1}=m_{2}=m_{3} \Rightarrow p=\frac{d^{\prime}}{2 d^{\prime}-d} \\
\sigma_{1}=\sigma_{2}=\sigma_{3} \Rightarrow d=0 \\
m_{1}=m_{2}=m_{3}=y \Rightarrow u_{i}=0 \Rightarrow s_{i}=\frac{\mathrm{I}}{2} \Rightarrow v=\frac{\mathrm{I}}{2}
\end{gathered}
$$

Donc, à strictement parler, il y aura fixation à une valeur $p=\frac{d^{\prime}}{2 d^{\prime}-d}$ :

si $d=$ o quelle que soit la valeur de $v$, c'est-à-dire si les deux homozygotes ont même valeur moyenne;

si $d \neq 0$ uniquement si nous retenons la moitié des pères.

Cependant, l'équation (I) admet en $p_{n}$ et $s_{i}$ d'autres solutions que celles déduites de l'identité (2). La solution théorique générale de l'équation (I) est difficile à obtenir.

Les résultats de calcul montrent en effet que, même pour $d \neq 0$ et une valeur de $v$ quelconque, on aboutit aussi, pratiquement, à une fréquence stable.

Quant à la stabilité d'un équilibre, on peut écrire :

$$
p_{n+1}=p_{n}+\frac{\mathrm{I}}{2 v} p_{n}\left(\mathrm{I}-p_{n}\right)\left[p_{n}\left(s_{1}-2 s_{2}+s_{3}\right)+s_{2}-s_{3}\right]
$$

$p_{n+1}$ sera supérieur à $p_{n}$ si

$$
\begin{gathered}
p_{n}\left(s_{1}-2 s_{2}+s_{3}\right)+s_{2}-s_{3}>0 \\
p_{n}\left(s_{1}-s_{2}\right)+\left(\mathrm{I}-p_{n}\right)\left(s_{1}-s_{3}\right)>0 \\
p_{n} \int_{u_{2}}^{u_{1}} g(u) \mathrm{d} u+\left(\mathrm{I}-p_{n}\right) \int_{u_{3}}^{u_{2}} g(u) \mathrm{d} u>0 \\
p_{n} \int_{u_{2}}^{u_{1}} g(u) \mathrm{d} u>\left(\mathrm{I}-p_{n}\right) \int_{u_{2}}^{u_{3}} g(u) \mathrm{d} u
\end{gathered}
$$


Relation vérifiée si $s_{1}<s_{2}<s_{3}$ ce qui entraîne $p_{n}<\frac{d^{\prime}}{2 d^{\prime}-d}$.

Donc la fréquence d'équilibre est une fréquence d'équilibre stable. Cependant cette condition est suffisante seulement et correspond à une majoration importante.

\section{2. -_ Possibilités d'oscillations}

On peut comprendre la réalisation d'oscillations entretenues d'une certaine amplitude pour $p$ avec les tailles de familles les plus grandes, dans la mesure où pour $\mathrm{N}$ assez grand, les distributions des descendances de mâles des trois génotypes $\mathrm{AA}, \mathrm{A} a$ et $a a$ sont pratiquement séparées.

Si tel est le cas et en posant $p_{0}=\frac{d^{\prime}}{2 d^{\prime}-d}$, on montre aisément que, pour $p<p_{0}$, les moyennes de ces descendances se classent dans l'ordre $m_{\mathrm{AA}}>m_{\mathrm{A} a}>m_{a a}$, l'ordre étant inversé si $p>p_{0}$.

Partant alors de $p<p_{0}$, si $v<p^{2}$, on choisira presque uniquement des pères AA; si $p^{2}+2 p(\mathrm{I}-p)>v>p^{2}$, on gardera tous les AA (proportion $p^{2}$ ) et une partie des $\mathrm{A} a$ (proporition $v-p^{2}$ ), de sorte que la fréquence à la génération suivante sera

$$
p^{\prime} \simeq \frac{I}{2}+\frac{p^{2}}{2 v}
$$

On aura

$$
p^{\prime}>p_{0} \quad \text { si } \quad \frac{\mathrm{I}}{2}+\frac{p^{2}}{2 v}>p_{0} \quad \text { ou } \quad v<\frac{p^{2}}{2\left(p_{0}-\frac{\mathrm{I}}{2}\right)}
$$

Dans le cas particulier où $p_{0}=\frac{\mathrm{I}}{2}(d=0)$, en posant $p=\frac{\mathrm{I}}{2}-h$, la condition d'oscillations d'amplitude stable $\left(p^{\prime}=\frac{\mathrm{I}}{2}+h\right.$ conduisant de nouveau à la fréquence $\frac{\mathrm{I}}{2}-h$ à la génération suivante par symétrie autour de $\left.\frac{\mathrm{I}}{2}\right)$ se traduit $\operatorname{par} v=\frac{p^{2}}{2 h}$.

On raisonnerait de même en partant de $p>p_{0}$.

On comprend également, à partir de ces exemples extrêmes, la nécessité d'une intensité de sélection suffisante.

\section{3. - Variations de la moyenne au cours des générations}

Ces quelques exemples montrent la possibilité, lors d'une sélection par test de descendance en présence d'un gène majeur avec surdominance, de fluctuations non monotones de la moyenne de la population au cours des générations pour le caractère sélectionné. Ces fluctuations seront la conséquence de celles de $p$, et pourront donc être limitées aux premières générations de sélection, ou entretenues régulièrement ; elles pourront, parfois, n'apparaître qu'après un certain nombre de générations.

Quant à l'amplitude de ces fluctuations de la moyenne, elle sera faible ou nulle si $d$ est petit ou nul. Dans le cas contraire, elle pourra ne pas être négligeable, mais sera cependant assez limitée en général. Ainsi, pour $\mathrm{N}=\mathrm{I} 00, d=0,5, d^{\prime}=\mathrm{I}, 5$ et $v=0$, Io la moyenne passe alternativement de 0,86 à 0,88 d'une génération à l'autre 
après quelques générations de sélection. Il faudra des valeurs $d$ et $d^{\prime}$ assez considérables pour rendre vraiment importante cette fluctuation de la moyenne, et lui donner, éventuellement, une valeur explicative pour certaines réponses inattendues observées vis-à-vis d'une sélection.

Reçu pour publication en février 1972.

\author{
SUMMARY \\ STABLE EQUILIBRIA OR EQUILIBRIA WITH PERIODIC OSCILLATIONS \\ OF ALLELIC FREQUENCIES IN SELECTION WITH PROGENY-TEST : \\ NUMERICAL EXAMPLES
}

On a series of numerical examples, we followed, over I 5 generations of selection for a quantitative trait, the evolution of the frequency of a gene $\mathrm{A}$ to a 2 - allele locus with overdominance for this trait, the residual variation being normally distributed. Selection is supposed to be exerted in one sex only by progeny-test with a fairly large number of mates, with random mating and non-overlapping generations.

There is always maintain of the two alleles, and in most cases a unique stable frequency is approached for A. On the other hand, in some situations, the possibility of permanent oscillations of this frequency is verified. These oscillations are the more important as selection intensity and family size are higher and the superiority of the heterozygous genotype is more marked. However, the corresponding fluctuations of the population mean are often of limited importance.

\title{
RÉFÉRENCES BIBLIOGRAPHIQUES
}

Hastings C. Jr., r955. Approximation for digital computers. Princeton University Press, Princeton, New Jersey, p. 186.

MÉrat P., I970. Rôle de l'intensité d'une sélection artificielle en présence de gènes à effet individuel important ou non. II. Sélection par familles, modèle déterministe. Ann. Génét. Sél. anim. 2, 429-447. 